\title{
Aplicación del método "Role-Playing" en prácticas de mantenimiento de aeronaves y su efecto en la motivación del alumno
}

\author{
Luis Miguel García-Cuevas ${ }^{1},{\text { Marcos } \text { Carreres }^{1} \text {, Andrés Tiseira }}^{1}$ y \\ Roberto Navarro ${ }^{1}$
}

${ }^{1}$ Universitat Politècnica de València

\begin{abstract}
The "Role-Playing" method has proved to be highly effective in the learning process, facilitating the understanding of theoretical contents and stimulating and motivating the students. In order to improve the student motivation, this teaching method has recently been introduced in the lab sessions of aircraft and jet engines maintenance taught in the Aerospace Engineering Bachelor Degree at the Universitat Politècnica de València. These lab sessions were originally taught with the classical approach consisting of a masterclass followed by an individual search on maintenance catalogues driven by the professor. The extent of the satisfaction and motivation of the student has been assessed by means of surveys conducted at the end of the lab sessions, showing promising results.
\end{abstract}

Keywords: Role-playing, teaching method, motivation, Aerospace Engineering, maintenance.

\footnotetext{
Resumen

El método de desempeño de roles o "Role-Playing" ha demostrado ser altamente efectivo en el proceso de enseñanza-aprendizaje, facilitando la comprensión de contenidos teóricos y estimulando y motivando al alumno. Para mejorar la motivación de los alumnos, este método didáctico ha sido introducido recientemente en las clases prácticas de mantenimiento de aeronaves y aerorreactores impartidas en la titulación de Grado en Ingeniería Aeroespacial de la Universitat Politècnica de València. Dichas prácticas eran impartidas originalmente con un enfoque clásico de clase magistral seguido de búsqueda de información en catálogos de mantenimiento de forma individual por parte del alumno y dirigida por el profesor.
} 
Aplicación del método "Role-Playing" en prácticas de mantenimiento de aeronaves y su efecto en la motivación del alumno

El grado de satisfacción y motivación de los mismos ha sido medido mediante las encuestas realizadas al finalizar las clases prácticas, mostrando buenos resultados.

Keywords: Role-playing, método didáctico, motivación, Ingeniería Aeroespacial, mantenimiento.

\section{Introducción y motivación}

Los alumnos que estudian cualquier carrera ingenieril adquieren sus conocimientos dentro de unas premisas asociadas a cumplimentar su formación más allá de una estricta relación entre la teoría aprendida con la práctica. Se destinan esfuerzos en vincular la enseñanza con la realización de proyectos, la invención de ideas, el trabajo en equipo, las relaciones humanas e, incluso, el respeto al medio ambiente y la ética profesional.

El programa teórico de las asignaturas suele complementarse con clases prácticas que permiten ayudar a comprender los conceptos explicados, favoreciendo la consecución exitosa del proceso de enseñanza-aprendizaje. Sin embargo, en ocasiones no se han aprovechado las sesiones prácticas para trasladar al alumno las situaciones laborales típicas que puede afrontar en su futuro profesional.

Se podrían plantear, por tanto, diversas técnicas y estrategias para mejorar el proceso de enseñanza-aprendizaje. Ello incluye, por ejemplo, ese traslado a las situaciones laborales típicas. La labor del docente, más que la de un mero transmisor de información, incluye la permamente reflexión sobre los métodos didácticos utilizados. El presente trabajo pretende demostrar cómo una práctica de laboratorio que inicialmente se concibió para introducir la terminología específica de mantenimiento aeronáutico ha sido actualizada haciendo uso de la técnica de desempeño de roles o "Role - Playing". De este modo, se espera una participación más activa por parte del alumno y se pretende mostrarle algunos de los aspectos del entorno profesional de un taller de mantenimiento aeronáutico. Por supuesto, en ningún momento se descuida la base teórico-práctica de la disciplina tratada.

La importancia de la técnica de enseñanza "Role-Playing" radica en establecer una relación más profunda entre la teoría, su práctica y la implementación de los conocimientos en el campo de trabajo real. De hecho, el "Role-Playing" es una técnica que se suele incluir en el llamado aprendizaje auténtico (Lombardi 2007). La técnica se usa en multitud de ámbitos, tanto para acercar la ingeniería a alumnos pre-universitarios (Fadali, Robinson y McNichols 2000) como para practicar la capacidad de empatía (Zowghi y Paryani 2003) u otros valores éticos (Prince 2006) en ingeniería. Incluso se aplica en la formación de profesionales del mantenimiento de aeronaves, con una excelente aceptación (Taylor y Robertson 1995). No obstante, algunos autores (Al-Ani y Yusop 2004) han mostrado que el alumnado no tiene una percepción unánime de que el "Role-Playing" ayude a consolidar lo aprendido. 
El siguiente apartado describe la práctica de laboratorio, tanto en su versión original como en su versión actualizada. Después, se comentan los resultados de la práctica desde el punto de vista de la motivación del alumno. Por último, se hace una discusión con las principales conclusiones y se comentan los trabajos futuros planteados por los autores.

\section{Descripción de la práctica}

La práctica de laboratorio se encuentra asociada a la teoría vista en la asignatura de Tecnología Aeroespacial, anticipando además contenido de la asignatura Mantenimiento de Aeronaves, ambas pertenecientes al Grado en Ingeniería Aeroespacial de la Universitat Politècnica de València. Esta práctica está asociada con el reconocimiento de elementos constructivos de los que comúnmente se componen las partes de aviones o de motores de aviación. El uso de manuales de mantenimiento ilustrados, en esta práctica, sirve como anticipo del contenido de la segunda asignatura.

Durante la práctica, se enseña a los alumnos a manejar un catálogo de piezas ilustrado (IPC) de un aerorreactor. Gracias a los manuales ilustrados de piezas aeronáuticas se consigue que cualquier elemento de un avión o motor en concreto pueda ser localizado y ubicado, permitiendo, incluso, saber cuál es su función. Asimismo, se hace una explicación introductoria sobre cómo se procede a dar mantenimiento a las distintas partes del motor.

La práctica se divide en dos partes claramente diferenciadas. La introducción teórica se realiza en primer lugar, siendo la misma tanto para la práctica tradicional como para aquélla para la que se ha utilizado el método de desempeño de roles. El contenido de dicha introducción es el siguiente:

- Se introduce el concepto de IPC y su nomenclatura habitual.

- Se explica cómo reconocer de forma fácil y sencilla las piezas de un motor o aeronave en el catálogo y cómo se procede a su búsqueda en el mismo.

- Se le enseña al alumno cómo interpretar la información aportada por el catálogo, como la función que posee la misma, su ubicación, el material con el que se encuentra confeccionada, etc.

- Se muestra al alumno el proceso que ha de seguir una pieza en el taller de mantenimiento, así como los requisitos fundamentales para que un taller pueda estar certificado para dar mantenimiento a dicha pieza.

Esta introducción teórica, de alrededor de una hora de duración, permite relacionar las piezas de un motor o aeronave con sus modos de funcionamiento, según lo visto en la asignatura de Tecnología Aeroespacial. Asimismo, se da una introducción a las políticas de mantenimiento empleadas en el sector aeronáutico, sirviendo de enlace con asignaturas posteriores como Mantenimiento de Aeronaves. 
Aplicación del método "Role-Playing" en prácticas de mantenimiento de aeronaves y su efecto en la motivación del alumno

\subsection{Método clásico}

Tras realizar la introducción teórica, los alumnos tienen acceso a un ordenador, el catálogo IPC de un aerorreactor y diversas piezas del mismo. De forma individual, los alumnos buscan información sobre las diversas piezas que tienen disponibles, intentando localizarlas en el catálogo de diversas formas y accediendo a la información detallada de las mismas. Durante todo este proceso, el profesor realiza una serie de preguntas a los alumnos sobre las piezas de las que disponen. Los alumnos tienen así una primera experiencia con el tipo de materiales utilizados en un taller de mantenimiento aeronáutico.

No obstante, en cursos anteriores se detectaron ciertos problemas durante la realización de la práctica: mientras un sector de los estudiantes mantenía el interés, gran parte del alumnado perdía la motivación tras realizar la búsqueda de un número reducido de piezas. Durante el curso 2015/16, y con el objetivo de mejorar este aspecto, se decidió implementar el método de desempeño de roles en 4 de los 6 grupos de la asignatura, como experiencia piloto, tal y como se explica en el siguiente apartado.

\subsection{Método de desempeño de roles}

La primera parte de la práctica, correspondiente a la explicación teórica y de manejo del catálogo IPC, se mantuvo mediante el enfoque tradicional. Finalizada esta fase, de alrededor de 1 hora, se organiza a los alumnos en grupos de 2 ó 3 estudiantes, facilitándoles una pieza real del aerorreactor por grupo junto con las siguientes indicaciones:

Sois operarios de mantenimiento de una importante aerolínea comercial. Acabáis de llegar a trabajar para relevar al grupo de compañeros del turno anterior. Durante el cambio, nadie reparó en que, encima de la mesa de trabajo, se ha quedado una pieza suelta de aerorreactor sin identificar.

Vuestro trabajo inmediato consiste en identificar la pieza en cuestión, dando su identificador o 'Part Number'. Una vez identificada, debéis inspeccionarla y evaluar si es apta para el servicio, si debe ser reparada o si debe ser retirada definitivamente del mismo, etiquetándola convenientemente. En caso de que la pieza no sea apta, debéis dar las razones por las que creéis que no lo es. 
Entre la información accesible a través del catálogo, se encuentran los boletines de servicio (service bulletins) de cada pieza, que contienen actualizaciones importantes para las mismas, tales como problemas existentes en el pasado, soluciones, reemplazos, etc.

Tened en cuenta que cualquier intervención innecesaria en la pieza supondrá grandes pérdidas económicas para la compañia, por lo que podéis recibir presiones de vuestros superiores en este sentido. Por el contrario, si una pieza necesitara una reparación o ser reemplazada y decidierais declararla apta para el servicio, se pondría en juego la seguridad de la aeronave, pudiendo acarrear pérdida de vidas humanas y responsabilidades penales en caso de accidente.

Además, a los alumnos se les facilita modelos de tarjetas utilizados en los talleres de mantenimiento reales para etiquetar las piezas como se pide. Dichas tarjetas siguen un código de colores de la siguiente manera:

Tarjeta verde: etiqueta de piezas esperando a ser reparadas. En ella se debe indicar la razón por la que la pieza debe ser reparada, y la tarea o tareas a llevar a cabo en la misma. Muchas veces, la información puede ser obtenida gracias a los mencionados service bulletins.

Tarjeta amarilla: identifica componentes aptos para su retorno al servicio.

Tarjeta roja: etiqueta piezas rechazadas, que no pueden volver a ser utilizadas en servicio. En las mismas se debe indicar el motivo del rechazo. Se puede ver un ejemplo de tarjeta roja en la Figura 1.

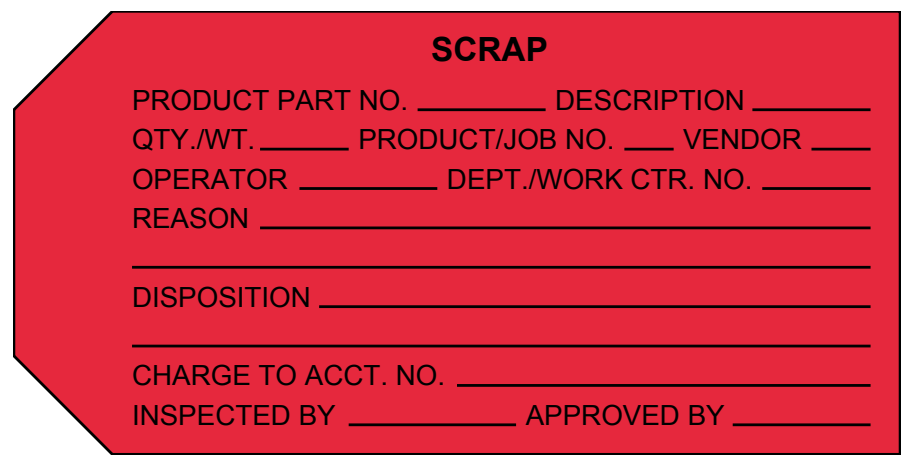

Fig. 1: Ejemplo de tarjeta de mantenimiento.

Una vez un grupo etiqueta convenientemente una pieza, se le facilita otra hasta completar un total de 4 en las 2 horas restantes de práctica. A lo largo de todo el proceso, el profesor monitoriza el trabajo de los alumnos y atiende sus dudas, desempeñando el rol de supervisor del turno de trabajo. 
Aplicación del método "Role-Playing" en prácticas de mantenimiento de aeronaves y su efecto en la motivación del alumno

\section{Resultados obtenidos}

Para evaluar el impacto del cambio de método didáctico utilizado en la práctica, se evalúa el nivel de motivación y satisfacción del alumno. Para ello, se utilizaron encuestas anónimas realizadas al finalizar la clase. En estas encuestas, se pide al alumno que valore con una puntuación de 1 a 5 los siguientes elementos:

1. Valoración global de la práctica

2. Contenidos

a) Es interesante

b) Está relacionada con los conocimientos teóricos de la asignatura

c) Aplica los conocimientos teóricos de la asignatura

3. Profesor

a) Domina la materia

b) Explica con claridad

c) Puntualidad: la práctica se inicia y finaliza a la hora prevista

4. Recursos

a) El material es el adecuado

b) Hay suficiente material

c) El laboratorio resulta confortable

5. Organización

a) La información de horarios y lugares es suficiente

b) La información de horarios está disponible a tiempo

Por último, el alumno puede dejar por escrito sus comentarios, observaciones y sugerencias. Estas encuestas son rellenadas de forma anónima mediante una aplicación web, de modo que el profesor no puede relacionar los resultados con un alumno en concreto.

Un total de 76 encuestas fueron introducidas por los alumnos. Los resultados de la valoración de la práctica se recogen en la Tabla 1:

10 de las encuestas presentaron comentarios adicionales, todas ellas por parte de alumnos del grupo de desempeño de roles. 
Tabla 1: Resultados de las encuestas

\begin{tabular}{ccc}
\hline Pregunta & Método clásico & Role-playing \\
\hline 1 & $4.167 \pm 0.140$ & $4.442 \pm 0.092$ \\
2.a & $4.458 \pm 0.118$ & $4.519 \pm 0.100$ \\
2.b & $4.125 \pm 0.207$ & $3.904 \pm 0.150$ \\
$2 . \mathrm{c}$ & $3.875 \pm 0.207$ & $3.942 \pm 0.140$ \\
3.a & $4.750 \pm 0.088$ & $4.769 \pm 0.075$ \\
3.b & $4.708 \pm 0.110$ & $4.712 \pm 0.083$ \\
3.c & $4.667 \pm 0.113$ & $4.750 \pm 0.076$ \\
4.a & $4.375 \pm 0.154$ & $4.615 \pm 0.087$ \\
4.b & $4.500 \pm 0.118$ & $4.442 \pm 0.113$ \\
4.c & $4.542 \pm 0.118$ & $4.538 \pm 0.084$ \\
5.a & $4.542 \pm 0.177$ & $4.731 \pm 0.098$ \\
5.b & $4.750 \pm 0.106$ & $4.827 \pm 0.065$ \\
\hline
\end{tabular}

Al analizar los resultados, no se encuentran diferencias significativas entre la valoración realizada por los alumnos sobre el profesor (preguntas 3.a, 3.b y 3.c), los recursos disponibles (preguntas 4.a, 4.b y 4.c) o la organización (preguntas 5.a y 5.b). Esto era de esperar, puesto que dichos aspectos no tendrían que cambiar entre ambos métodos. Asimismo, el hecho de que las diferencias en la valoración de los profesores no sean significativas da indicios de que éstos no han cometido un sesgo importante a la hora de impartir la docencia, lo que podría haber modificado la valoración final de la práctica. Sí se puede ver una ligera mejora en la valoración global de la práctica (pregunta 1), dándose la mejor valoración en el caso de la práctica con método de desempeño de roles. Es interesante comprobar que las respuestas a las preguntas sobre si la práctica es interesante, está relacionada con los contenidos teóricos de la asignatura y aplica los conocimientos teóricos de la asignatura no presentan diferencias estadísticamente significativas. Es posible, no obstante, tener una idea de por qué los alumnos acabaron dando una valoración global mayor a la práctica realizada con desempeño de roles analizando los comentarios dados por los mismos. Se han seleccionado los siguientes comentarios:

Ha resultado muy interesante que la práctica haya sido más interactiva y que hayamos podido "trabajar" como responsables de manteniemiento. También, el buscar en la guía en inglés ha resultado útil porque en un futuro tendremos que utilizar documentación en inglés y con lenguaje técnico cuando trabajemos en mantenimiento.

Ha resultado interesante para acercarnos un poco al ámbito profesional, algo a lo que no estamos acostumbrados en las clases teóricas. 
Aplicación del método "Role-Playing" en prácticas de mantenimiento de aeronaves y su efecto en la motivación del alumno

La práctica ha resultado esta vez más amena, dinámica, e interactiva debido a la colaboración de distintos grupos para resolver una serie de problemas.

Analizando los comentarios de los alumnos, se encuentra que valoran positivamente el trabajo en grupo y la simulación de un ambiente laboral. En opinión de los autores de este trabajo, es probablemente este último aspecto el que más valoran y que mayor motivación produce. Los alumnos ven poco claro su futuro laboral, no siempre tienen claras todas las posibilidades de trabajo a las que pueden acceder gracias a su titulación y muchas veces no son conscientes de que están en proceso de convertirse en profesionales altamente cualificados con una gran cantidad de competencias útiles adquiridas. El acercamiento a las actividades típicas del mundo profesional hace que sean más conscientes de todo esto, lo que redunda en su nivel de motivación.

\section{Conclusiones}

Para la realización de este trabajo, se ha partido de una práctica con la que ya se tenía cierta experiencia y se ha adaptado al método de desempeño de roles, en un intento de aumentar el nivel de motivación de los alumnos al ubicarles en un simulacro de ambiente laboral. Para ello, los alumnos fueron divididos en grupos que realizaron la práctica de la forma clásica y grupos que la realizaron con el nuevo método. Tanto las apreciaciones subjetivas percibidas por los profesores como las encuestas rellenadas por parte de los alumnos indican un mayor nivel de satisfacción y motivación de los alumnos. Analizando los comentarios realizados por estos últimos, parece que lo que más valoran es el acercamiento al mundo profesional llevado a cabo con este enfoque de clase práctica.

Los autores de este trabajo plantean continuar con él en el futuro, estudiando la repercusión de la aplicación de este métood docente en los resultados de aprendizaje del alumnado. Se pretende realizar un test al finalizar la práctica, tanto al grupo de control como al grupo de alumnos que la realicen con el nuevo método, además de un segundo test un mes más adelante. En dichas pruebas, se le preguntará a los alumnos sobre el contenido de la práctica. Partiendo de la hipótesis de que un mayor nivel de motivación favorece el proceso de enseñanza-aprendizaje, los autores esperan encontrar diferencias significativas en los resultados de dichos tests.

\section{Referencias bibliográficas}

Al-Ani, Ban y Norazlin Yusop (2004). "Role-playing, group work and other ambitious teaching methods in a large requirements engineering course". En: Engineering of Computer-Based Systems, 2004. Proceedings. 11th IEEE International Conference and Workshop on the. IEEE, págs. 299-306. 
Fadali, M Sami, Mike Robinson y K McNichols (2000). "Teaching engineering to K-12 students using role playing games". En: ASEE Annual Conf., American Society for Engineering Education, Washington, DC. Citeseer.

Lombardi, Marilyn M (2007). "Authentic learning for the 21st century: An overview". En: Educause learning initiative 1.2007, págs. 1-12.

Prince, Robert H (2006). "Teaching engineering ethics using role-playing in a culturally diverse student group". En: Science and Engineering Ethics 12.2, págs. 321-326.

Taylor, JC y MM Robertson (1995). The effects of Crew Resource Management (CRM) training in airline maintenance: Results following three year's experience. Inf. téc. NASA.

Zowghi, Didar y Suresh Paryani (2003). "Teaching requirements engineering through role playing: Lessons learnt". En: Requirements Engineering Conference, 2003. Proceedings. 11th IEEE International. IEEE, págs. 233-241. 